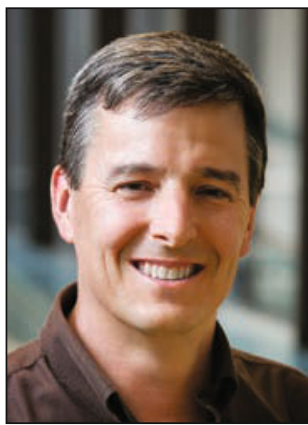

Tohn A. Rogers, Northwestern University, has received the 2018 Materials Research Society (MRS) Medal "for pioneering contributions to materials for diverse classes of bio-integrated electronic systems." Rogers will be recognized during the Awards Ceremony at the 2018 MRS Fall Meeting in Boston.

Rogers is the Louis Simpson and Kimberly Querrey Professor at Northwestern University, with appointments in materials science and engineering, biomedical engineering, neurological surgery, electrical and computer engineering, mechanical engineering, and chemistry. He is also the founding director of the endowed Center on Bio-Integrated Electronics. He received BA and BS degrees in chemistry and in physics from The University of Texas at Austin, and his PhD degree in physical chemistry from the Massachusetts Institute of Technology.

Rogers's scientific and engineering accomplishments fall mainly into four categories: tissue-integrated electronic/ microfluidic systems as wireless platforms for continuous, clinical-quality assessment of physiological health and as interventional/diagnostic tools for treating diseases of the heart and brain; cellular-scale optoelectronics as ultraminiaturized implants for optogenetic studies of neural function and for electrotherapy of neurological disorders; bioresorbable electronics as temporary implants for sensing, drug delivery, and neuromodulation; and three-dimensional electronic networks as interfaces to cells and growing tissues.

In each case, operation has been validated in studies and trials; several are now in commercial use. The materials range from soft elastomers, to shape memory polymers, semiconductor nanomaterials, colorimetric responsive chemistries, and heterogeneous combinations of these with more established materials. Rogers's research spans materials synthesis and fundamental properties, to integration strategies, device design, and system engineering.

Rogers is the recipient of a MacArthur Fellowship; the Lemelson-MIT Prize; election to the National Academy of Sciences, the National Academy of Engineering, and the American Academy of Arts \& Sciences; and election to the College of Fellows of the American Institute for Medical and Biological Engineering. He has published more than 650 papers, is the inventor on more than 100 patents and patent applications, and has co-founded several startup companies.

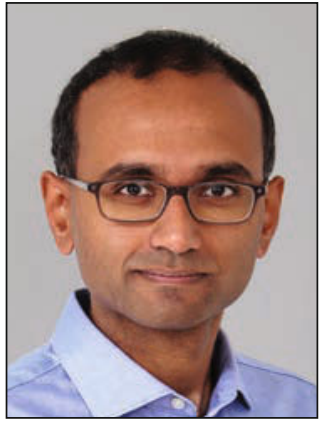

\section{Aaswath P. Raman to receive the inaugural MRS Nelson "Buck" Robinson Science and Technology Award for Renewable Energy}

\footnotetext{
$\mathrm{A}$ aswath P. Raman, University of California, Los Angeles, will receive the inaugural Materials Research Society (MRS) Nelson "Buck" Robinson Science and Technology Award for Renewable Energy, which recognizes a student, postdoc, or other early-career professional through five years following the highest degree attained, for the development of novel sustainable solutions for the realization of renewable sources of energy.
}

Raman is an assistant professor in the Department of Materials Science and Engineering at the University of California, Los Angeles. He is the co-founder and chief scientific officer of SkyCool Systems, a startup commercializing radiative sky cooling technology based on research that he originated. He previously was an assistant professor of electrical and systems engineering at the University of Pennsylvania, and was a research associate and postdoctoral scholar at Stanford University.

Raman's research spans metamaterials and nanophotonics to heat transfer, energy systems, and computational methods, with a particular focus on enabling new energy technologies. He received his $\mathrm{PhD}$ degree in applied physics from Stanford University. He is the recipient of the Sir James Lougheed Award of Distinction from the Government of Alberta, Canada, the SPIE Green Photonics Award for his work on solar-cell research, and the Stanford Postdoctoral Research Award. In recognition of his breakthroughs in developing radiative sky cooling, in 2015, Raman was named one of MIT Technology Review's Innovators Under 35 (TR35) as an Energy Pioneer.

MRS acknowledges the generosity of Sophie Robinson for endowing this award in memory of her father, Nelson "Buck" Robinson. 